\title{
Test Arrangement for the W7-X HTS-Current Lead Prototype Testing
}

\author{
W. H. Fietz ${ }^{1}$, S. Drotziger ${ }^{1}$, S. Fink ${ }^{1}$, M. Heiduk ${ }^{1}$, R. Heller ${ }^{1}$, A. Kopmann ${ }^{1}$, C. Lange ${ }^{1}$, \\ R. Lietzow ${ }^{1}$, T. Möhring ${ }^{1}$, P. Rohr ${ }^{1}$, T. Rummel ${ }^{2}$, M. Süßer $^{1}$ \\ Karlsruhe Institute of Technology, Karlsruhe, Germany \\ Max-Planck-Institut für Plasmaphysik, Teilinstitut Greifswald, Greifswald, Germany
}

The Karlsruhe Institute of Technology (KIT) is responsible for the design, construction and testing of the high temperature superconductor (HTS) current leads for the stellarator Wendelstein 7-X (W7-X) which is presently under construction at the Greifswald branch of the Max-Planck-Institute for Plasma Physics. Because the W7-X current leads are mounted with the warm end at the bottom a special test cryostat has been built and is attached to the main cryostat of the TOSKA facility of KIT. Two prototypes of these current leads are tested in this cryostat under W7-X relevant conditions. The test conditions with respect to cryogenic, control, current supply, data acquisition and quench detection of the test setup for the prototype test at TOSKA is described. The performance of the MC plug used to power the HTS current leads is described in detail.

\section{INTRODUCTION}

CuRR temperature level to the low temperature operation level of the coils (typically $4.5 \mathrm{~K}$ ). After the successful construction and test of the ITER HTS CL demonstrator at $68 \mathrm{kA}$ which has been developed and built by the Forschungszentrum Karlsruhe (now KIT) in collaboration with the Centre Recherches en Physique des Plasmas, Switzerland in the frame of an EU Fusion Technology task [1], KIT has taken responsibility for design, construction and test of the HTS-CL for the Stellarator W7-X and the satellite tokamak JT-60SA. The CL consists of three parts, a copper heat exchanger (HEX), a HTS module and the CL cold end. The HEX is cooled by $50 \mathrm{~K}$ helium at the cold end side. To this end of the HEX the HTS module is connected that is conduction cooled only and leads the current to the CL cold end that is actively cooled with $4.5 \mathrm{~K} \mathrm{He}$. Details can be found in [2-4].

To test the CLs after production, a dedicated test bed "CuLTKa" (Current Lead Test facility Karlsruhe) is under construction at KIT. However, CuLTKa will not be operational before 2011. Therefore it was decided to perform the tests of the prototype CLs for W7-X in TOSKA. Because the W7-X current leads are operated in "upside-down" condition (warm end at the bottom), TOSKA has to be adapted to allow a test under such special conditions.

This paper gives an overview of the test ensemble with two current leads connected by a short-circuit busbar and it describes the preparation of TOSKA for the W7-X prototype current lead test with respect to cryogenics, current supply, control \& data acquisition, electronics and quench detection. In addition basic data of the test setup are given. Details of the test results with respect to the CL prototype cannot be shown here due to space constraints but can be found in [4].

This work was performed within the European Fusion Technology Program of the Karlsruhe Institute of Technology, and under contract 03/100/4500149540 between the Max-Planck-Institut für Plasmaphysik and the Karlsruhe Institute of Technology 


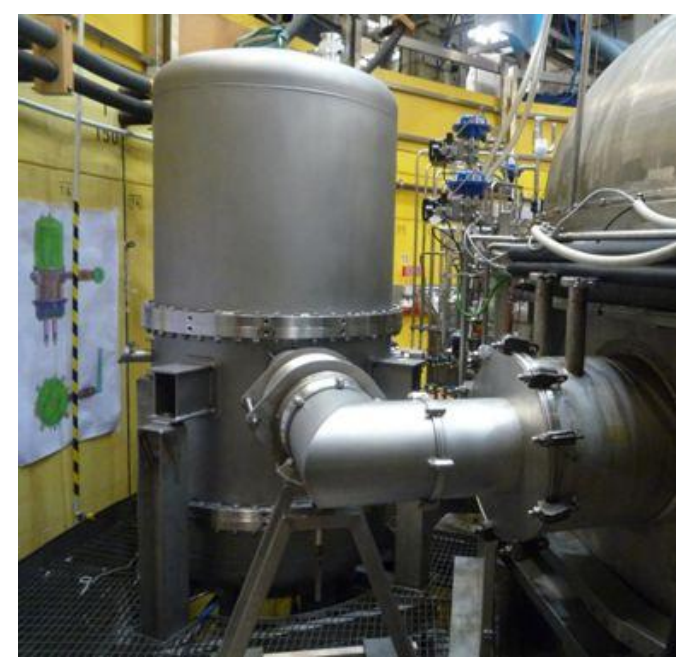

Fig. 2. Test cryostat attached to the large TOSKA cryostat with transfer line needed for helium feeding. The current supply to feed the current leads is connected from below.

\section{Current LeAd Test Ensemble}

TOSKA is able to integrate four current leads in normal orientation, but the installation of current leads in upside down orientation is not possible. As a consequence a test cryostat which has been already fabricated for the later use in CuLTKa was attached as a satellite cryostat to TOSKA. The two W7-X CL prototypes are mounted in the lower part of the test cryostat which is supported in a special frame (see Fig. 1) for installation. At the top the two $\mathrm{CL}$ prototypes are connected by a short-circuit busbar fabricated by IPP (Max-Planck-Institut für Plasmaphysik) using the W7-X conductor. Clamp contacts with indium foils are used to connect the CLs with the short-circuit busbar.

Because the highest geometric level of this satellite cryostat is higher then the $\mathrm{LN}_{2}$ shield of TOSKA, a helium cooled thermal shield has been integrated in this cryostat. A special designed transfer line feeds $4.5 \mathrm{~K}$ helium for short-circuit busbar and CL cold end, and the $50 \mathrm{~K}$ helium for the $\mathrm{CL}$ heat exchanger to this satellite cryostat. Fig. 2 shows the test cryostat attached to the large TOSKA cryostat together with the He transfer line.

\section{He Flow Scheme And Cryogenics}

The TOSKA facility is connected to the $2 \mathrm{~kW}$ refrigerator by two separate transfer lines (details see Fig. 3).

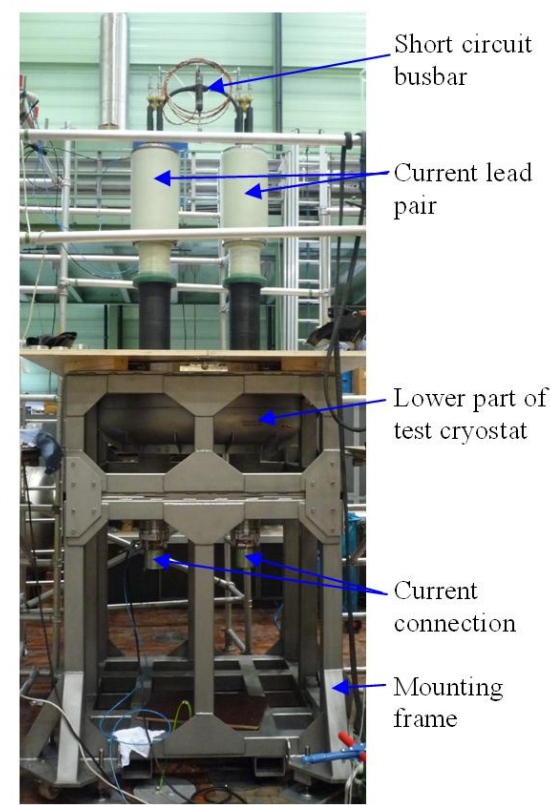

Fig.1 Test ensemble with current lead prototype pair connected by a shortcircuit busbar installed in the lower part of test cryostat. 
Transfer line I is used to cool down the complete test configuration with helium from room temperature to $20 \mathrm{~K}$. The helium used during operation is supplied by the $2 \mathrm{~kW}$ refrigerator through transfer line II, expanded through a JouleThomson valve and liquefied into the control dewar (B250). He-pumps and heat exchangers of the secondary cooling loop are submerged in the liquid helium bath of the dewar. The secondary cooling loop is filled and pressurized by the $2 \mathrm{~kW}$ refrigerator at the suction side of the pumps, in doing so the helium in the forced flow circuit is kept at a supercritical pressure level of approx. 4.5 bar. The busbar system is forced-flow cooled with liquid or supercritical helium in the secondary cooling loop either by one of the two installed piston pumps or by a centrifugal pump. The heat exchangers of the HTS CLs are operated with helium in the temperature range between $20 \mathrm{~K}$ and $80 \mathrm{~K}$ directly from the $2 \mathrm{~kW}$ refrigerator through transfer line I. The advantage of the separated lines is that the control dewar can be filled with liquid helium while the test arrangement is cooled down separately and the forced flow operation of the test configuration can start immediately by the pumps to reach the operation temperature of $4.5 \mathrm{~K}$.

In standby mode during nights and weekends, the whole test arrangement is directly cooled by the $2 \mathrm{~kW}$ refrigerator through the dewar B250 without use of the helium pumps.

Under normal test conditions, the system is designed to release no helium into the atmosphere. However, unexpected severe fault conditions can force the release of helium gas through safety valves and burst disks to the atmosphere. For all fault conditions, e.g. electrical power outage or refrigerator shut down, the system switches over to a safe mode where the system can remain unattended without damage.

\section{POWER SUPPLY AND CURRENT FEEDING}

The $50 \mathrm{kA}$ power supply of TOSKA was used for the CL prototype test. The maximum test current was $20 \mathrm{kA}$ which is just above the maximum current of the W7-X CLs of $18.2 \mathrm{kA}$. Water cooled high-current copper cables were used as room temperature feeders to feed the current to an aluminum connection part located next to the CL current connection. To match the operation condition of W7-X, Multi-Contact (MC) [5] plugs of W7-X were used in TOSKA, too. An aluminum MC plug with copper strand connections is visible in Fig. 4 together with the lower end of the $C L$ prototypes at the top. To ensure that the MC plugs will not warm up under high-current operation, five additional water cooling rings were installed for each $\mathrm{CL}$.

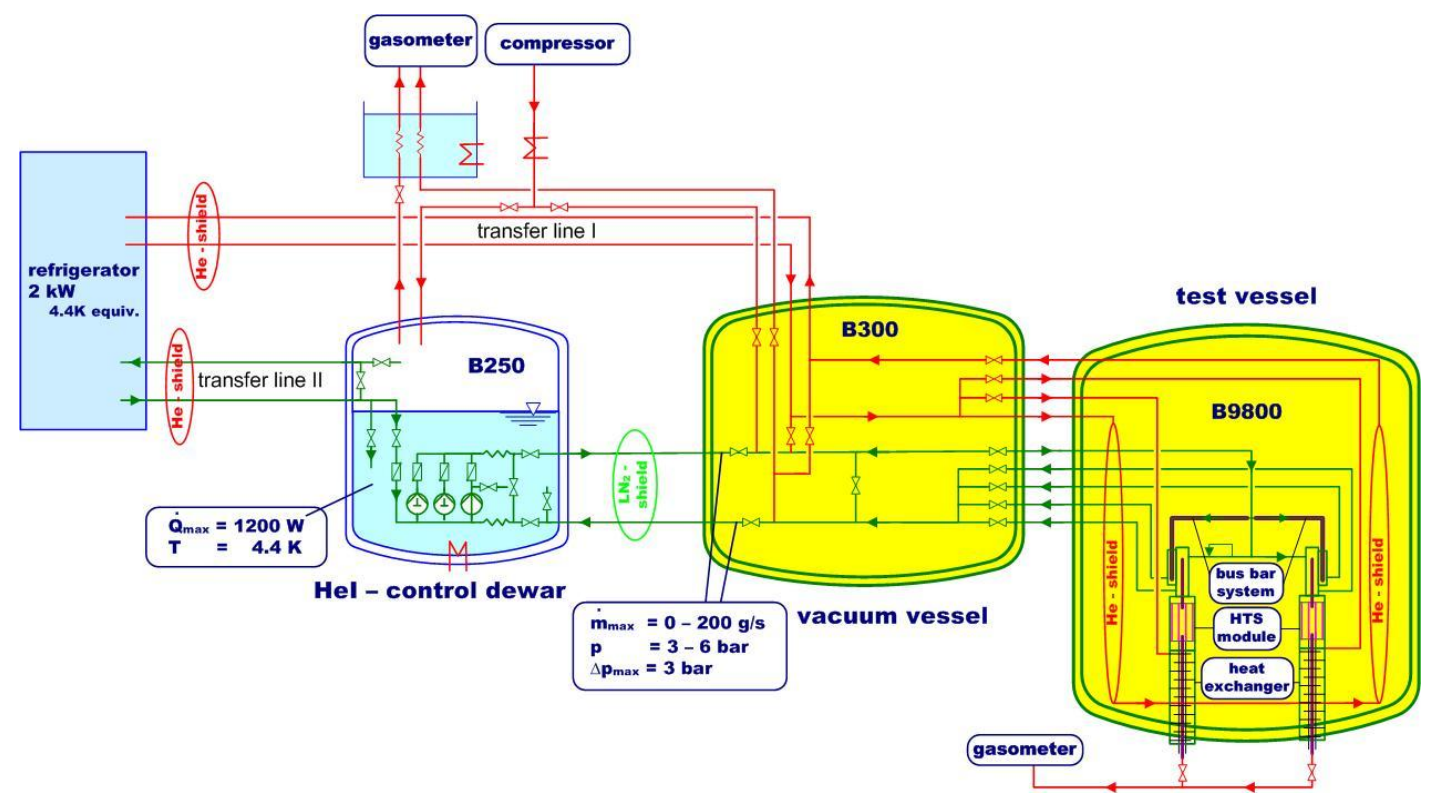

Fig. 3. Test cryostat B9800 attached to the large TOSKA cryostat B300 with a transfer line needed for heliumhelium feeding. The current supply to feed the current leads is connected from below. 


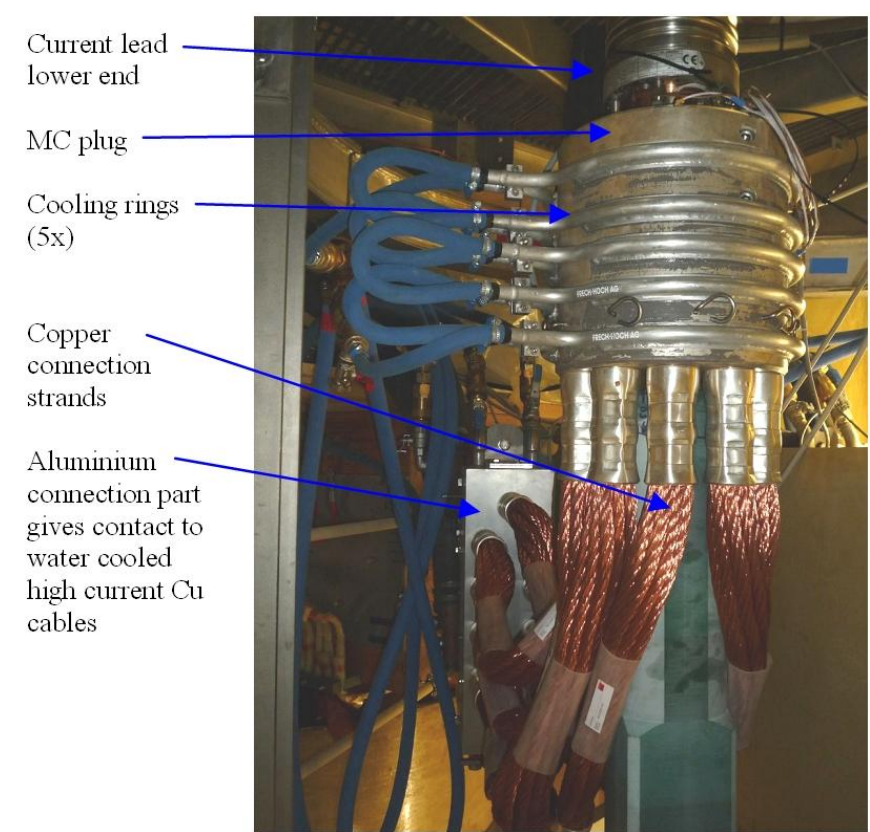

Fig. 4. Multi -Contact plug at lower end of W7-X current lead prototype with five water cooling rings and the copper connection strands.

In this CL test we had no magnet installed. As a consequence the energy in the system was rather low and the invertor mode of the power supply was fast enough to ramp down the current in case of problems. Therefore we did not use fast discharge in case of a quench but invertor mode discharges, only.

\section{CONTROL AND DATA ACQUISITION}

The data acquisition has been designed as a modular and hierarchical system with three layers: Programmable logic controllers and PC-based measurements in the field level, data storage and monitoring in the second layer. The third layer is dedicated to the first data analysis and data distribution.

A SIMATIC PCS 7 control system ensures the safe operation of the complex cryosystem of TOSKA. Pressure and pressure drop are directly measured by the PCS 7 system using Endress \& Hauser (PMP71 \& PMD75) pressure transducers with Profibus connection.

Most temperatures are measured with temperature sensors directly mounted inside the He pipes to avoid temperature offsets due to bad thermal coupling. He mass flow is calculated using pressure, pressure drop and temperature.

There are two standalone PC measurement systems using PXI hardware and running with the Windows XP operating system. Measured physical quantities are voltages, current and temperatures on the device under test as well as mass flow of cooling water and other temperatures from the facility's infrastructure. Furthermore, calculated quantities like thermal power loss on segments of He pipes or cool down rates are available.

These items and selected sensor values from the PCS7 system are recorded with data rates between one and ten Hertz into the primary MS SQL database. A dedicated Live Display allows one to view selected sensors directly from this primary database. While the primary database is dedicated mainly to recording the data, a mirrored database serves for data distribution. The advanced data extraction infrastructure (ADEI) has been developed for the first inspection of the recorded data. ADEl provides a fast web-based graphical navigation through the complete experimental data by using Web 2.0 (AJAX) technologies. Selected views can be saved as bookmarks in the browser, and be downloaded in a number of formats for further analysis [6].

\section{ELECTRONICS AND QUENCH DETECTION}

In case of fast discharges of magnets or during high voltage tests the voltages and sensor signals from the CLs are on high voltage level. As a consequence isolation amplifiers are used to measure voltages. To measure temperatures, isolation transducers with integrated free-of-ground current sources are used.

For quench detection (QD) the TOSKA QD system was equipped with two quench detectors using separated voltage lines for the HTS module and the short-circuit busbar connecting the CL. The standard voltage level was 10 $\mathrm{mV}$ with $100 \mathrm{~ms}$ integration time. For some special experiments the voltage level was increased to $50 \mathrm{mV}$ (details see [4]). 


\section{OPERATION EXPERIENCE}

Fig. 5 gives an overview of the W7-X CL prototype test with cool down, experimental campaign and warm up.

The CL was cooled down with $\mathrm{He}$ from the refrigerator, starting at $\sim 300 \mathrm{~K}$. The temperature was decreased by $\sim 10 \mathrm{~K} / \mathrm{h}$ during daytime. At night the temperature was held constant. After reaching approx. $10 \mathrm{~K}$ the cooling was changed to a forced flow mode using dedicated He pumps installed in our control cryostat. Initial verifications test at low temperature were performed followed by tests with temperature increases at the heat exchanger at zero current. The current operation is visible in Fig. 5 demonstrating a maximum current of $20 \mathrm{kA}$. A long term operation of 6 hours at $18.2 \mathrm{kA}$ is directly visible (see Monday, before warm up was started). The CL could be operated smoothly. Details of these experiments will be published elsewhere (e.g. [4]).

\section{Vill. Performance of mC Plug}

Before current operation was started the performance of the specially designed MC plugs was not clear because the plug and the copper strands attached to this plugs are air cooled only. In case of a heat generation by these plugs the produced power would directly heat the CL lower end and add losses to the CL. To balance such a possible heat production we added the five cooling rings visible in Fig. 4 to recool the lower end of the CL.

In the experiment even at $18.2 \mathrm{kA}$ we found only a small heat generation at these MC plugs. The temperature of the plugs reached only $31^{\circ} \mathrm{C} @ 18.2 \mathrm{kA}$ even when the water cooling was switched off completely. The copper strands reached temperatures of $29^{\circ} \mathrm{C} @ 18.2 \mathrm{kA}$ in regions were the strands are air cooled and approx. $35^{\circ} \mathrm{C}$ in the region where they are wrapped with plastic (see Fig. 4 and Fig. 6).

To determine the contact resistance of single copper strand connectors to the Multi-Contact plug the voltage over several strands under a current of $9 \mathrm{kA}$ was measured. All voltages were in the range between $1.7 \mathrm{mV}$ and $2.2 \mathrm{mV}$. The average contact resistance was $2.1 \mu \Omega$.

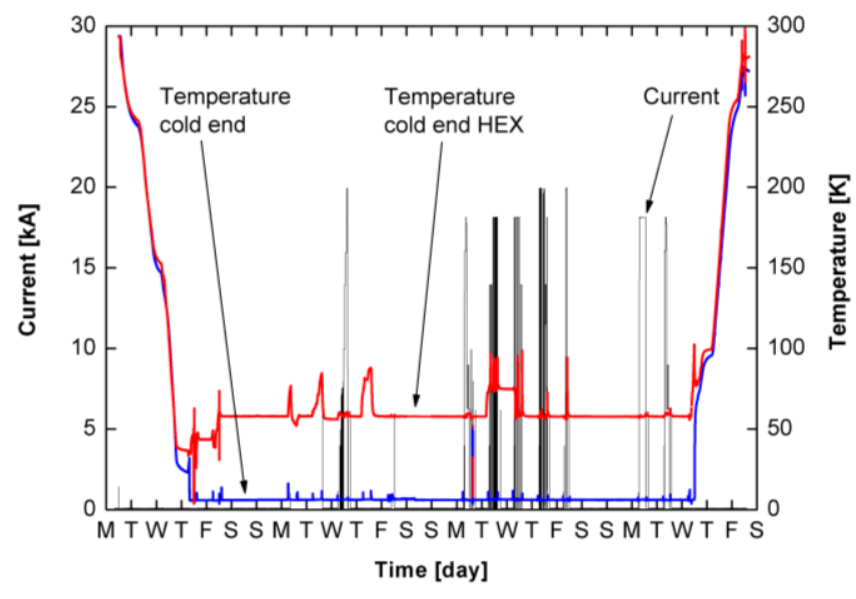

Fig. 5. Temperature at the cold end of the CL and at the cold end of the HEX during cool down, experiment and warm up. In addition the current Fig. 6. Temperature of the Multi Contact plug at lower end of W7-X current operation is shown.

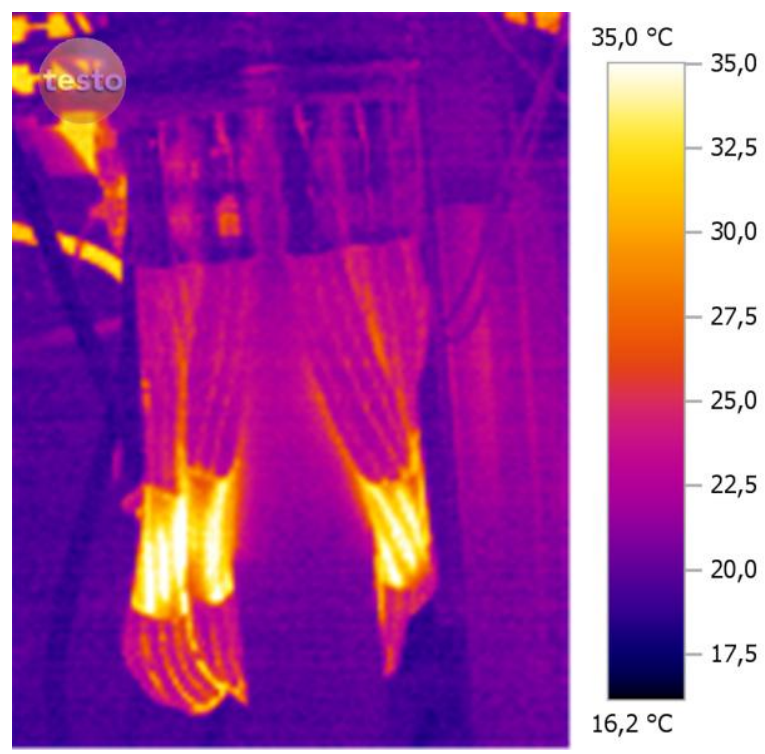

lead prototype with five water cooling rings and the Copper connection strands at $20 \mathrm{kA}$. 


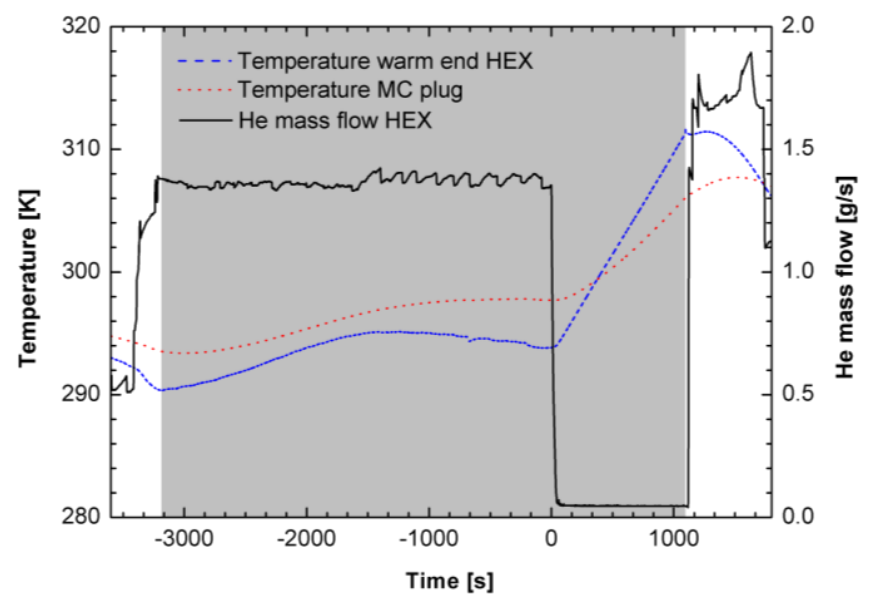

Fig. 7. Temperature at current lead heat exchanger (HEX) warm end (dashed line) and MC plug (dotted line) together with He-flow through HEX (solid line). The CL has been operated at $18.2 \mathrm{kA}$ in the shaded area.

As a consequence of these excellent values it was decided to switch off the water cooling for all further experiments in these W7-X CL tests

\section{Performance of MC Plug during Loss of Flow}

During the experimental campaign the $\mathrm{CL}$ was tested with a maximum current of $18.2 \mathrm{kA}$ (shaded region in Fig. 7) with no water cooling at the MC plug. To simulate a loss of $50 \mathrm{~K} \mathrm{He}$ flow through the heat exchanger, this flow was stopped (see Fig. 7, moment of He stoppage adjusted to $t=0$ ). Due to a temperature increase at the HTS module (not shown in Fig.7) the HTS part quenched after approx. $1060 \mathrm{~s}$ and the current was reduced to zero (end of shaded region in Fig.7). The experiment shows clearly that temperature of the $\mathrm{MC}$ plug does not excess $35^{\circ} \mathrm{C}$ even in the case of a 18 minute loss of $50 \mathrm{~K}$ He flow under the full current of $18.2 \mathrm{kA}$.

The MC plug specially designed for W7-X is fully functional and allows operating under all possible operation scenarios.

\section{SUMmaRY}

The experimental setup to determine the performance of the HTS CLs specially designed for W7-X was described. The installation with respect to cryogenics, control, current supply, data acquisition and quench detection was functional and performed as expected.

Special attention was paid to the MC plug specially designed for W7-X. Even in the worst case scenario of a 17 minutes loss of $50 \mathrm{~K}$ helium cooling of the copper HEX of the $\mathrm{CL}$, the MC plug temperature does not exceed a temperature of $35^{\circ} \mathrm{C}$.

\section{ACKNOWLEDGMENT}

Many thanks to the TOSKA crew and the $2 \mathrm{~kW}$ refrigerator crew for their professional job.

\section{REFERENCES}

[1] R. Heller et al., "Development of High Temperature Superconductor Current Leads for $70 \mathrm{kA}$ " IEEE Trans. on Appl. Supercond. vol 12 no. 1 pp. 1285-1288, 2002.

[2] W.H. Fietz, R. Heller, A. Kienzler, and R. Lietzow, "Status of HTS current leads for WENDELSTEIN 7-X and JT60SA" Fusion Engineering and Design 84 (2-6), pp. 776-779, 2009.

[3] W.H. Fietz, R. Heller, A. Kienzler, and R. Lietzow, "High Temperature Superconductor Current Leads for WENDELSTEIN 7-X and JT-60SA" IEEE Trans. on Appl. Supercond. vol 19 no 3, pp. 2202-2205, 2009.

[4] R. Heller et al., "Test Results of the High Temperature Superconductor Prototype Current Leads for Wendelstein 7-X" presented at Applied Superconductivity Conference, Washington, USA, Aug 1 - 6, 2010.

[5] Multi-Contact AG, see http://www.multi-contact.com/

[6] S. Chilingaryan, A. Beglarian, A. Kopmann, S. Voecking, "Advanced data extraction infrastructure: Web based system for management of time series data" J. of Physics: Conference Series,. 219 042034, 2010. 